\title{
Key dynamical results from the SAMI Galaxy Survey
}

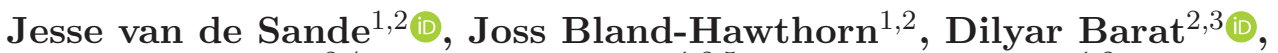 \\ Sarah Brough ${ }^{2,4}$, Julia J. Bryant ${ }^{1,2,5}$, Scott M. Croom ${ }^{1,2}$, \\ Luca Cortese ${ }^{2,6}$ and the SAMI Galaxy Survey collaboration \\ ${ }^{1}$ Sydney Institute for Astronomy, School of Physics, A28, The University of Sydney, \\ NSW, 2006, Australia \\ email: jesse.vandesande@sydney.edu.au \\ ${ }^{2}$ ARC Centre of Excellence for All Sky Astrophysics in 3 Dimensions (ASTRO 3D), Australia \\ ${ }^{3}$ Research School of Astronomy and Astrophysics, Australian National University, \\ Canberra ACT 2611, Australia \\ ${ }^{4}$ School of Physics, University of New South Wales, NSW 2052, Australia \\ ${ }^{5}$ Australian Astronomical Optics, AAO-USydney, School of Physics, \\ University of Sydney, NSW 2006, Australia \\ ${ }^{6}$ International Centre for Radio Astronomy Research, The University of Western Australia, \\ 35 Stirling Highway, Crawley WA 6009, Australia
}

\begin{abstract}
We present an overview of recent key results from the SAMI Galaxy Survey on the build-up of mass and angular momentum in galaxies across morphology and environment. The SAMI Galaxy survey is a multi-object integral field spectroscopic survey and provides a wealth of spatially-resolved, two-dimensional stellar and gas measurements for galaxies of all morphological types, with high-precision due the stable spectral resolution of the AAOmega spectrograph. The sample size of $\sim 3000$ galaxies allows for dividing the sample in bins of stellar mass, environment, and star-formation or morphology, whilst maintaining a statistical significant number of galaxies in each bin. By combining imaging, spatially resolved dynamics, and stellar population measurements, our result demonstrate the power of utilising integral field spectroscopy on a large sample of galaxies to further our understanding of physical processes involved in the build-up of stellar mass and angular momentum in galaxies.
\end{abstract}

Keywords. galaxies: evolution - galaxies: kinematics and dynamics - galaxies: stellar content

\section{Introduction}

Studying how galaxies build-up their mass and angular momentum across time is key to understanding the varying properties observed across the Hubble-Sequence in the local Universe (Tinsley 1980). This requires detailed measurements of both the stellar populations and stellar kinematic properties that provide a fossil-record of how galaxies were assembled. A strong link between stellar mass and angular momentum over time is expected as galaxy luminosity or stellar mass is strongly correlated with the kinematic ratio of ordered to random stellar motion (e.g., Illingworth 1977; Davies et al. 1983). While these results, which were based on single-slit spectroscopy, pioneered the field, a major step forward was facilitated by the introduction of visible-light integral field spectrographs (IFS) such as SAURON (Bacon et al. 2001). The kinematic properties of galaxies could now be measured in two dimensions rather than along a single axis.

The SAURON survey (de Zeeuw et al. 2002) and ATLAS ${ }^{3 \mathrm{D}}$ survey (Cappellari et al. 2011) produced the first volume-limited samples of early-type galaxies with 
two-dimensional (2D) stellar kinematic maps. These surveys led to a new understanding towards the stellar angular momentum distribution of early-type galaxies and its connection to visual morphology (Krajnović et al. 2011; Emsellem et al. 2011). However, in order to form a complete picture of the build-up of stellar mass and angular momentum over time, a sample with galaxies across the full Hubble-Sequence is required.

The CALIFA survey was the first IFS campaign to observe a galaxy sample with a large range in visual morphology (Sánchez et al. 2012). Although early results revealed some peculiar properties of low-mass spiral galaxies (Falcón-Barroso et al. 2015)), the total sample sized used for extracting stellar kinematics is still relatively low $(N \sim 300)$. Multiobject IFS surveys such as SAMI (Sydney-AAO Multi-object Integral field spectrograph; Croom et al. 2012) and the SDSS-IV MaNGA Survey (Sloan Digital Sky Survey Data; Mapping Nearby Galaxies at APO; Bundy et al. 2015) now makes it possible to analyse large $(N>1000)$ numbers of galaxies with a broad range in morphology, mass, and environment.

This review focuses on the key dynamical results from the SAMI Galaxy Survey and how these results bring us closer to understanding the build-up of mass and angular momentum in galaxies across morphology and environment. Some of the results described are closely linked to findings made by the SAURON, ATLAS ${ }^{3 \mathrm{D}}$, CALIFA, and the MaNGA surveys. These were reviewed separately at this Symposium, in particular by Shude Mao, Ling Zhu, and Michele Cappellari. Furthermore, the reader is referred to Cappellari (2016) for a combined review of all the different kinematic measurement techniques used for this work.

\section{The SAMI Galaxy Survey}

SAMI is a multi-object integral field spectrograph mounted at the prime focus of the $3.9 \mathrm{~m}$ Anglo Australian Telescope (AAT; Croom et al. 2012). The instrument has 13 IFUs, or hexabundles (Bland-Hawthorn et al. 2011; Bryant et al. 2014) that can be deployed across a $1^{\circ}$ diameter field of view; each IFU covers a $\sim 15 "$ angle on the sky. All 819 (793 plus 26 sky fibres) fibers are fed into the AAOmega dual-beamed spectrograph (Sharp et al. $2006)$. The grating for the blue $(580 \mathrm{~V})$ and red $(1000 \mathrm{R})$ arms resulted in a resolution $\mathrm{R}_{\text {blue }} \sim 1808$ at $4800 \AA$, and $\mathrm{R}_{\text {red }} \sim 4304$ at $6850 \AA$ (Scott et al. 2018 ), and a wavelength coverage of $3750-5750 \AA$ and $6300-7400 \AA$. Observations were executed using a seven-point dither pattern to create data cubes with 0." 5 spaxel size (Sharp et al. 2015; Allen et al. 2015).

The SAMI Galaxy Survey has observed $~ 3000$ galaxies (Bryant et al. 2015) between redshifts $0.004<z<0.095$, with galaxy stellar mass $\mathrm{M}_{\star}=10^{8}-10^{12} M_{\odot}$, and galaxy environment ranging from the field to group and clusters. Targets were selected from the Galaxy and Mass Assembly (GAMA) G09, G12 and G15 regions (Driver et al. 2011), and eight high-density cluster regions sampled within radius $R_{200}$ (Owers et al. 2017). Reduced data-cubes, gas and stellar kinematic data products for 1559 galaxies in the GAMA fields are available at: https://datacentral.org.au/, as part of the first and second SAMI Galaxy Survey data release (Green et al. 2018; Scott et al. 2018).

\section{The build-up of mass and angular momentum in galaxies across morphology and environment}

In this new era of large multi-object IFS surveys, different observing strategies and target selection criteria are employed in order to optimally achieve the science goals set-out at the start of the survey. Yet, with the wealth of data that is now available to the community, one can wonder where different surveys are complementary or different. The uniqueness of the SAMI Galaxy Survey arises from a combination of four features: i) a large range in visual morphology, ii) a sample size of $N \sim 3000$ that is large enough to 
(a)

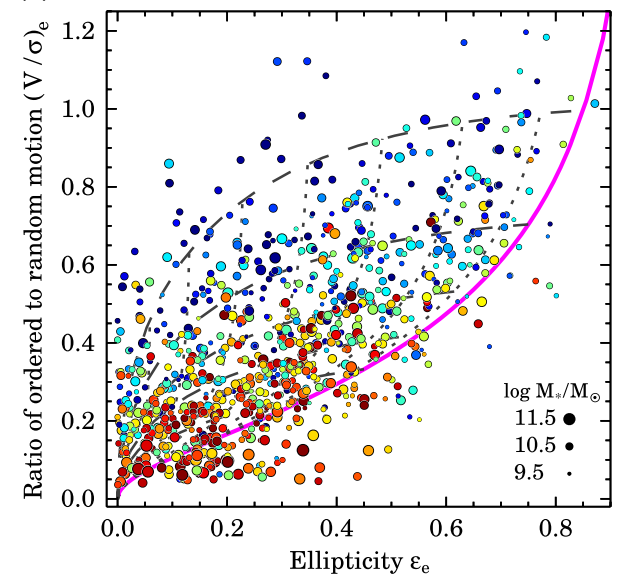

(b)

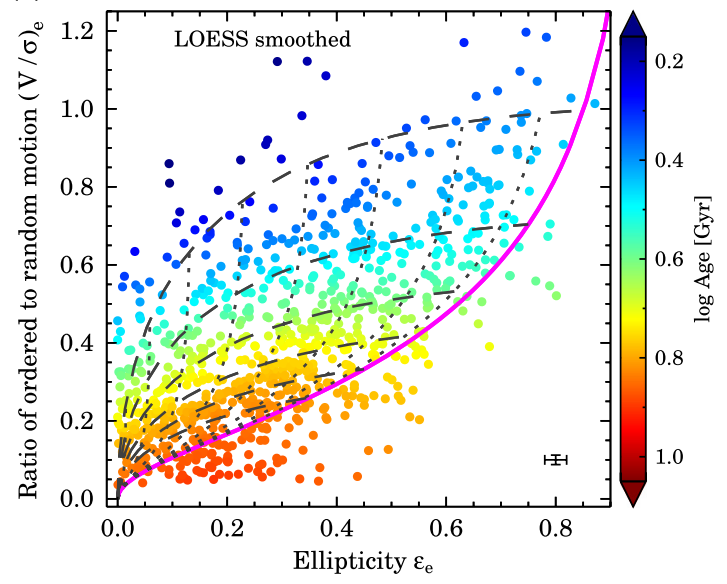

Figure 1. Ordered to random stellar motion $(V / \sigma)_{\mathrm{e}}$ and observed ellipticity $\varepsilon_{\mathrm{e}}$ (from van de Sande et al. 2018, Fig. 2). The colour coding reveals the luminosity-weighted stellar age within one effective radius. In panel a), the symbol size indicates the total stellar mass of the galaxy of each individual galaxy. In panel b) we use the LOESS smoothing algorithm to recover the mean underlying trend in mean stellar age. We show theoretical predictions for axisymmetric rotating oblate spheroids, observed edge-on, as the solid magenta line. The dotted lines highlight different viewing angles from edge-on (magenta line) to face-on (towards zero ellipticity). Galaxies with different intrinsic ellipticities ( $\varepsilon_{\text {intr }}=0.85-0.35$ from top to bottom) are indicated by the dashed lines. The mean stellar age of galaxies follow the lines of constant intrinsic ellipticity remarkably well.

split galaxies by mass, environment, morphology or star-formation rate, iii) a large range in environment from field to groups and massive clusters, iiii) a stable spectral resolution of $R=1808$ in the blue and an exquisite resolution in the red $R=4304$. In the following, we will highlight several key results that employ these unique features of the survey.

Utilising SAMI's large range galaxy morphology. Cortese et al. (2016) give quantitative evidence that galaxies of all morphological types lie on a plane that relates stellar mass, specific angular momentum, and the stellar-light distribution as quantified by the Sérsic index. Both the stellar and gas specific angular momentum $j_{\star}\left(<R_{\mathrm{e}}\right)$ correlate strongly with stellar mass, with a linear slope (coefficient $\sim 0.64$ ) that is remarkably close to that of analytical models $(2 / 3)$. The scatter in the $j_{\star}-M_{\star}$ relation is related to the Sérsic index, which implies that this relation is a good statistical predictor of galaxy morphology. From the tight relation between the Sérsic index and the stellar spin parameter $\lambda_{R}$, Cortese et al. (2016) show that fast-rotating early-type and late-type galaxies form a continuous class, kinematically connecting pure discs to bulge-dominated systems.

By analysing the stellar population properties of galaxies in the $(V / \sigma)_{\mathrm{e}}-\varepsilon_{\mathrm{e}}$ diagram, van de Sande et al. (2018) reveal that there is a relation between the mean age of a galaxy and the intrinsic ellipticity (see Fig. 1). This discovery extends beyond the classical paradigm where "disks are young" and "bulges are old", as it reveals a gradual transformation from thin to thick spheroids as the stellar population grows older. The same relation is recovered in different stellar mass regimes, even at low stellar masses $\left(\log \left(M_{\star} / M_{\odot}\right)<10.25\right)$ where the fraction of classical dispersion dominated bulges is low. Furthermore, the relation remains when the sample is split by visual morphology into early and late-type galaxies. The intrinsic ellipticity is also a more accurate, dynamical and physically motivated equivalent of the bulge-to-disk ratio measurements derived from imaging (e.g., Lange et al. 2016). 
(a)

(b)

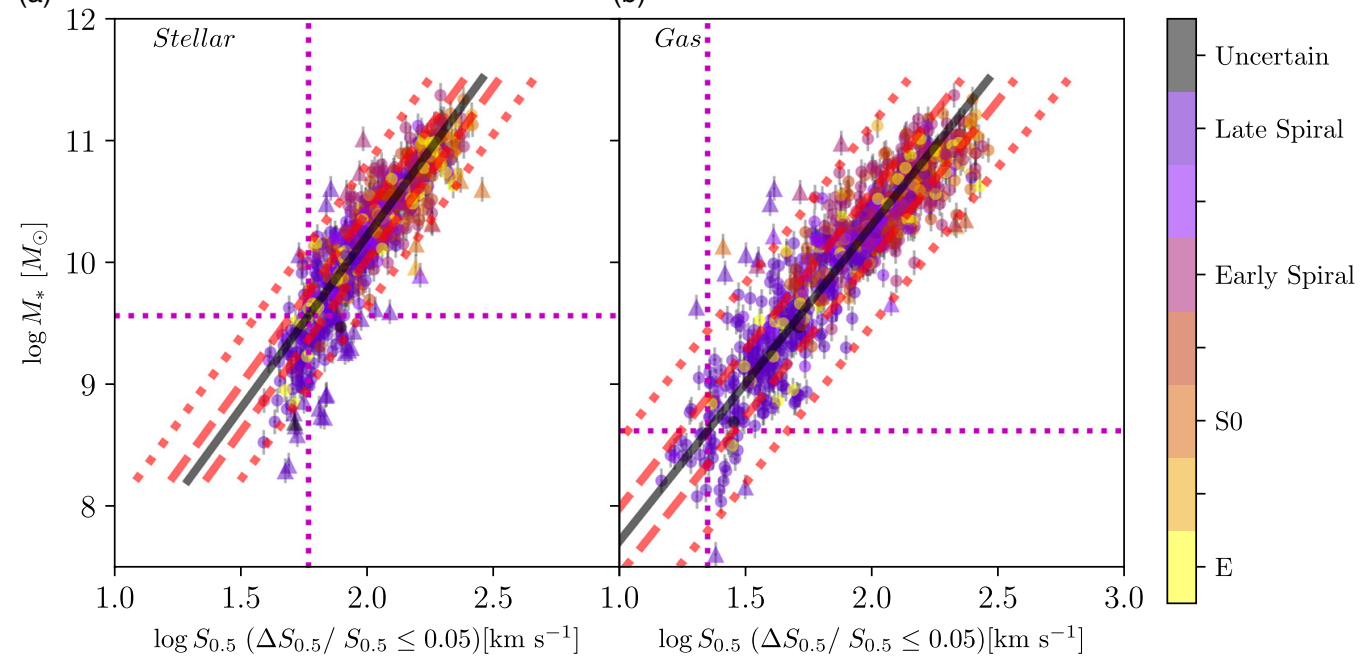

Figure 2. $\log \left(M_{\star} / M_{\odot}\right)-\log S_{0.5}$ scaling relations for stars (panel a) and gas (panel b) (from (Barat et al. 2019), Fig. 4). Data are colour coded according to their visual morphological type as indicated by the side bar. The magenta horizontal and vertical dotted lines show the location where the distribution of points deviate from a linear relation. For the stars, we find a best-fit relation of $\log S_{0.5}=0.35 \pm 0.01 \log \left(M_{\star} / M_{\odot}\right)-1.60 \pm 0.10$, a slightly steeper relation of $\log S_{0.5}=0.39 \pm 0.01 \log \left(M_{\star} / M_{\odot}\right)-1.99 \pm 0.05$ derived from the gas kinematics. The solid black line indicates these best-fit linear relations, whereas the red dashed and dotted lines show 1 and 3 RMS distance from these best-fit lines. These relations apply to both early-type and late-type galaxies, and show smaller scatter than either the Tully-Fisher relation $\left(\log \left(M_{\star} / M_{\odot}\right)-\log V_{\text {rot }}\right)$ for late types, or the Faber-Jackson relation $\left(\log \left(M_{\star} / M_{\odot}\right)-\log \sigma_{\mathrm{e}}\right)$ for early types.

Utilising SAMI's spectral resolution. The $S_{0.5}$ parameter, derived from the velocity and velocity dispersion such that $S_{\mathrm{K}}=\sqrt{K V_{\text {rot }}^{2}+\sigma^{2}}$, is a useful quantity that can unify the stellar and gas kinematic measurements of galaxies with all morphological types onto a single dynamical scaling relation (Weiner et al. 2006; Kassin et al. 2007; Cortese et al. 2014). The $\log \left(M_{\star} / M_{\odot}\right)-\log S_{0.5}$ relation is explored in detail in Barat et al. (2019) who use the SAMI Galaxy Survey to probe this relation down to low-stellar masses $\left(\log \left(M_{\star} / M_{\odot}\right)<9\right.$; see Fig. 2). At these low stellar masses, SAMI's instrumental resolution is critical for deriving accurate velocity dispersions. Both stellar and gas $\log \left(M_{\star} / M_{\odot}\right)-$ $\log S_{0.5}$ relations deviate from a linear relation towards low-stellar mass. However, the sample limits occur at different stellar masses for the gas and stellar measurements, which implies that the apparent non-linearity in the relation is not physical. Barat et al. (2019) also empirically test the impact of changing the value of $\mathrm{K}$ in the $S_{K}$ parameter. By measuring the scatter in $\log \left(M_{\star} / M_{\odot}\right)-\log S_{\mathrm{K}}$ as a function of $K$ for both gas and stars, they find that the optimal value of $K$ is between 0.2 and 0.7 for samples containing only early-type galaxies or mixtures of early and late-type galaxies. This confirms the validity of using $K=0.5$ as the optimal value for $S_{K}$.

A well-defined, stable spectral resolution is also critical for the analysis of high-order stellar kinematic parameters $h_{3}$ and $h_{4}$ that quantify the skewness and kurtosis of the stellar line-of-sight velocity distribution. Using a high $\mathrm{S} / \mathrm{N}$ subsample from the SAMI Galaxy Survey, van de Sande et al. (2017) analyse the high-order kinematic $h_{3}$ versus $V / \sigma$ signatures of galaxies. For galaxies in similar regions of the $\lambda_{R_{\mathrm{e}}}-\varepsilon_{\mathrm{e}}$ diagram, these highorder kinematic signatures can be distinctly different, revealing new physical structures that cannot be identified from using $V$ and $\sigma$ alone. These results are compared with prediction from the hydrodynamical simulations presented in Naab et al. 2014. Simulated 
galaxies show different $h_{3}-V / \sigma$ relations depending on whether a galaxy had experienced predominantly gas-rich or gas-poor mergers in the past. The predictions for gas-rich mergers match the observational signatures well. However, we do not find evidence for a significant population of fast-rotating galaxies without a stellar disk as predicted from gas-poor mergers. These results suggest that the majority of fast-rotating galaxies were formed in gas-rich mergers and that gas-poor mergers are unlikely to be a dominant formation path.

Utilising SAMI's large sample size. An analysis of the kinematic misalignment of the gas and stellar rotation fields is presented in Bryant et al. (2019). By comparing the kinematic position angles of the velocity fields, 11 per cent of galaxies are classified as misaligned $\left(\triangle P A>30^{\circ}\right)$. The misalignment fraction is highest ( $45 \pm 6$ per cent) for galaxies with early-type morphology or high Sérsic index, but only $5 \pm 1$ per cent for late-type galaxies. However, no significant correlation is found between the misalignment fraction and stellar mass, colour or local environment. To constrain the origin of gas in galaxies, these results are compared to predictions of different theoretical scenarios. Analysis of the gas/star misalignment distribution find insufficient late-type galaxies settling to be counter-rotating compared to predictions if the gas was accreted at all angles. Instead, the distribution supports simulations in which the gas in late-type galaxies is accreted with an angular momentum axis aligned with that of the stars, or torqued towards existing gas at a radius higher than the SAMI field of view. In contrast, early-type galaxies have misalignment distributions consistent with the gas being accreted from any angle (Davis \& Bureau 2016).

The large number of IFS galaxy observations are also ideally suited for comparing resolved two-dimensional stellar dynamical measurements to simulations. In van de Sande et al. (2019) we directly compare structural, resolved dynamical, and stellar population measurement of mock galaxies from the EAGLE (Schaye et al. 2015; Crain et al. 2015) and HYDRANGEA (Bahé et al. 2017), HORIZON-AGN (Dubois et al. 2014), and MAGNETiCUM (see Dolag et al. in prep and Hirschmann et al. 2014) simulations to observations from SAMI, ATLAS ${ }^{3 D}$, CALIFA, and the MASSIVE (Ma et al. 2014) surveys. The main goal is to identify key areas of success and tension, not to determine which simulations provides the "best" or closest match to observations. Considerable effort is devoted to making sure that simulated measurement methods match the observational methods as closely as possible, and simulated mock samples are constructed that match the observed stellar mass distribution for the combined IFS sample. The following structural quantities are compared, either as a function of stellar mass or compared to one another: $R_{\mathrm{e}}$, $\varepsilon_{\mathrm{e}}, \sigma_{\mathrm{e}}, M_{\mathrm{dyn}},(V / \sigma)_{\mathrm{e}}$, mean stellar age, and $\varepsilon_{\text {intr }}$. We show one example in Fig. 3 where observed and simulated galaxies are compared in the $(V / \sigma)_{\mathrm{e}}-\varepsilon_{\mathrm{e}}$ plane. The overall comparison between observational and simulated data has highlighted several areas where the simulations could be improved, such as the need for better modelling to improve the vertical disk structure. Yet these results also demonstrate the enormous improvement of cosmological simulations in recent years.

Utilising SAMI's large range in environment. The formation of slow-rotating galaxies has received considerable attention over the last decade (see review by Cappellari 2016). One of the outstanding questions is the role of the environment in this process and whether a kinematic morphology-density relation exists. Brough et al. (2017) focus on the 8 massive clusters within the SAMI Galaxy Survey to address this question. They measure the fraction of slow-rotating galaxies within the early-type population and find a strong increase in the slow rotator fraction towards high stellar mass, mean environmental overdensity, and towards the centre of clusters. However, after controlling for stellar mass, they no longer find a significant difference between galaxies in high-overdensities 

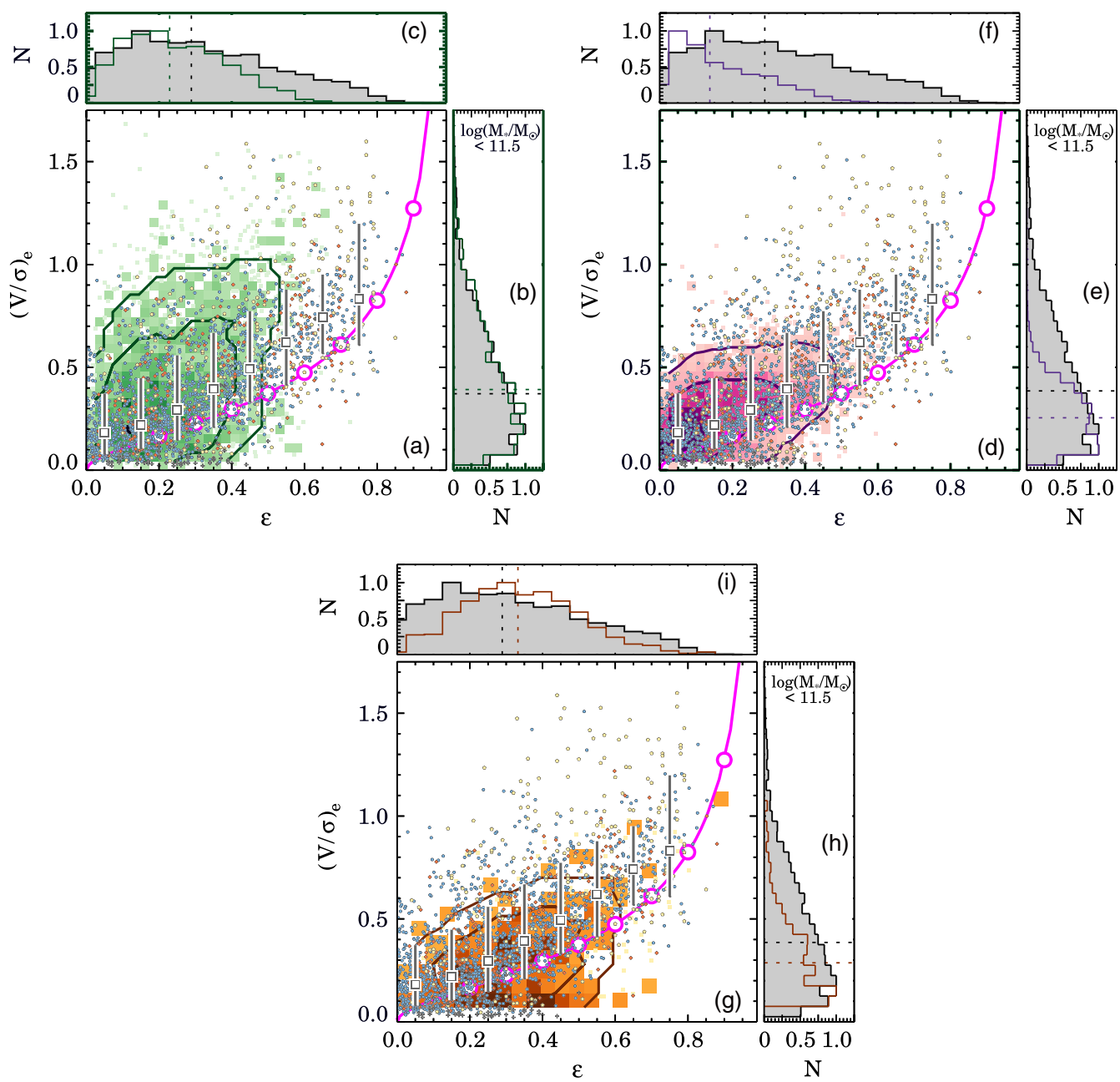

Figure 3. Comparing observed and simulated galaxies in the $(V / \sigma)_{\mathrm{e}}-\varepsilon_{\mathrm{e}}$ diagram (adopted from van de Sande et al. 2019, Fig. 8). The small squares indicate the density of galaxies from the simulations where darker colour shows a higher density of galaxies (panel a) EAGLE and HYDRANGEA in green; panel d) HORIZON-AGN in purple; panel g) MAGNETICUM in brown). The contours enclose 68 and 95 percent of the simulated data, We show observational data from the SAMI Galaxy Survey as blue circles, ATLAS ${ }^{3 \mathrm{D}}$ as orange diamonds, CALIFA as yellow pentagons, and MASSIVE as grey pluses. Median of the observed sample in mass bins are shown by the grey and white squares, whereas the vertical lines indicate the 16th and 84th percentile. The magenta line shows the theoretical prediction similar to Fig. 1. In each top and side panel, we show the distribution of the simulations (coloured) and observational data (black), with the dotted lines indicating the median. Data from the MASSIVE survey is not included in this panel. The shape of the distribution of EAGLE and HYDRANGEA data is steeper than observations, with few simulated galaxies extending beyond $\varepsilon_{\mathrm{e}}>0.6$ and $(V / \sigma)_{\mathrm{e}}>0.5$. For HORIZON-AGN, the shape of the distribution is broadly correct, but $\varepsilon_{\mathrm{e}}$ and $(V / \sigma)_{\mathrm{e}}$ are significantly lower than as observed. MAGNETICUM predictions closely follow the shape of the observed distribution, yet at fixed $\varepsilon_{\mathrm{e}}$ galaxies with high $(V / \sigma)_{\mathrm{e}}$ are not found.

and low-overdensities. Yet, at fixed environmental overdensity massive galaxies are more likely to be slow rotators. Thus, the primary driver for the kinematic morphology-density relation in the centre of clusters is caused by the increasing fraction of massive galaxies towards high-density environment (see also Veale et al. 2017 and Greene et al. 2017). 


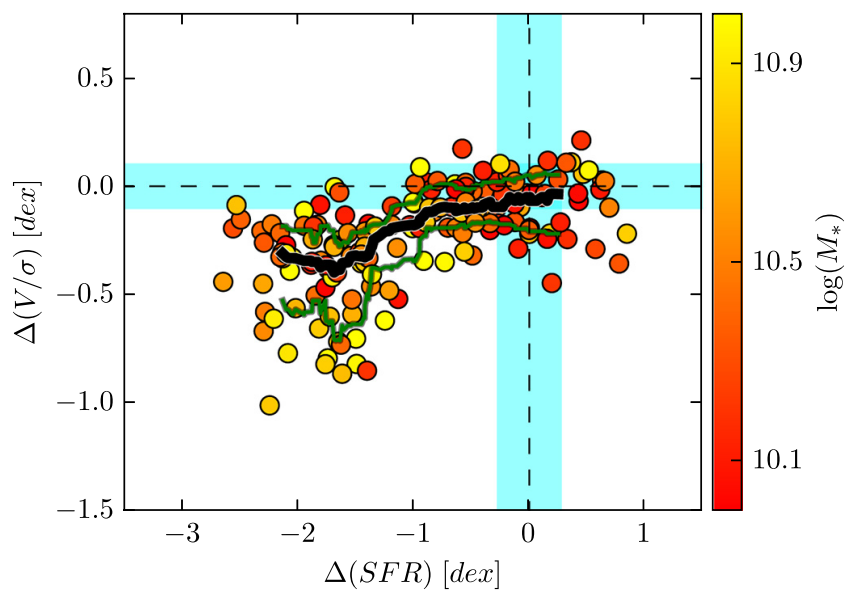

Figure 4. Offsets in stellar $(V / \sigma)_{\mathrm{e}}$ and $S F R$ for satellite galaxies as compared to main SFR sequence, high $(V / \sigma)_{\mathrm{e}}$ central galaxies (From Cortese et al. 2019, Fig. 3). The variation in $(V / \sigma)_{\mathrm{e}}$ and $S F R$ for the central galaxy sample is given by the cyan bands (1- $\sigma$ standard deviation). For the observed SAMI Galaxy Survey data, the black line shows the running median, with the 20 and 80 percentile ranges for $\Delta(V / \sigma)_{\mathrm{e}}$ in bins of $\Delta$ SFR. Data are colour coded by stellar mass. Satellite galaxies show the largest difference in SFR as compared to centrals, whereas $(V / \sigma)_{\mathrm{e}}$ decreases the strongest for satellites that have already quenched and reached the red sequence $(\triangle S F R<-1)$.

By targeting galaxies in the GAMA regions, the SAMI Galaxy Survey has access to the high-quality group catalogues (G3Cv1, Robotham et al. 2011) that were build from the deep and highly-complete GAMA spectroscopy. Cortese et al. (2019) use this group catalogue to study if satellite quenching is accompanied by a change in the dynamical properties of those satellites as quantified $V / \sigma$. By comparing satellite galaxies to a control sample of central, star-forming and high $V / \sigma$ galaxies, they show that galaxies undergo little dynamical change during their quenching phase (see Fig. 4). With the same analysis on a sample of galaxies from the EAGLE simulations, Cortese et al. (2019) find similar results, where satellite galaxies show a larger change in $\Delta$ SFR than in $\Delta V / \sigma$. However, when the change in SFR and $V / \sigma$ is studied by comparing the satellite's property at $z \sim 0$ with the properties in the last simulation snapshot before infall, the change in $V / \sigma$ is even less. This shows that the analysis with observational data can only provide an upper limit for the real amount of transformation experienced by galaxies during their satellite phase.

\section{Conclusion}

We present eight key dynamical results from the SAMI Galaxy Survey that utilise different features that make the survey unique.

1) We show that galaxies of all types lie on a plane relating mass, angular momentum and stellar-light distribution (Cortese et al. 2016), 2) and that there is a relation between stellar population age and intrinsic ellipticity (van de Sande et al. 2018). 3) By measuring the $S_{0.5}$ parameter, we demonstrate that we bring together the gas and stellar kinematic measurements of galaxies with all morphologies onto a single dynamical scaling relation (Barat et al. 2019). 4) With high-order stellar kinematic measurements, we determine that galaxies with similar $\lambda_{R_{\mathrm{e}}}-\varepsilon_{\mathrm{e}}$ values can show different $h_{3}-V / \sigma$ signatures, which can be linked to their assembly history by comparing to zoom-in simulations (van de Sande et al. 2017). 5) A comparison of the kinematic misalignment of the gas and stellar rotation fields shows that the presence of misaligned gas is a strong function of morphology 
(Bryant et al. 2019). 6) We also compare IFS observations to large cosmological simulations, which highlights several areas for improvement, but also demonstrate the vast advances in cosmological simulations in recent years (van de Sande et al. 2019). 7) By analysing galaxies in the SAMI Cluster regions, we find that the fraction of slow rotators correlates correlates more strongly with stellar mass than with environment (Brough et al. 2017. 8) By studying smaller groups in the GAMA regions, we find that satellite galaxies undergo little structural change during their quenching phase (Cortese et al. 2016).

\section{Acknowledgements}

It is a great pleasure to thank the organisers and attendees for an enjoyable conference, and to my colleagues in the SAMI Galaxy Survey team for making this work possible.

The SAMI Galaxy Survey is supported by the Australian Research Council Centre of Excellence for All Sky Astrophysics in 3 Dimensions (ASTRO 3D), through project number CE170100013, the Australian Research Council Centre of Excellence for All-sky Astrophysics (CAASTRO), through project number CE110001020, and other participating institutions. The SAMI Galaxy Survey website is http://sami-survey.org/. JvdS is funded under JBH's ARC Laureate Fellowship (FL140100278). JJB acknowledges support from and ARC Future Fellowship (FT180100231).

\section{References}

Allen, J. T., Croom, S. M., Konstantopoulos, I. S., et al. 2015, MNRAS, 446, 1567

Bacon, R., Copin, Y., Monnet, G., et al. 2001, MNRAS, 326, 23

Bahé, Y. M., Barnes, D. J., Dalla Vecchia, C., et al. 2017, MNRAS, 470, 4186

Barat, D., D'Eugenio, F., Colless, M., et al. 2019, MNRAS, 487, 2924

Bland-Hawthorn, J., Bryant, J., Robertson, G., et al. 2011, Optics Express, 19, 2649

Brough, S., van de Sande, J., Owers, M. S., et al. 2017, ApJ, 844, 59

Bryant, J. J., Bland-Hawthorn, J., Fogarty, L. M. R., et al. 2014, MNRAS, 438, 869

Bryant, J. J., Owers, M. S., Robotham, A. S. G., et al. 2015, MNRAS, 447, 2857

Bryant, J. J., Croom, S. M., van de Sande, J., et al. 2019, MNRAS, 483, 458

Bundy, K., Bershady, M. A., Law, D. R., et al. 2015, ApJ, 798, 7

Cappellari, M., Emsellem, E., Krajnović, D., et al. 2011, MNRAS, 413, 813

Cappellari, M. 2016, ARAESA, 54, 597

Cortese, L., Fogarty, L. M. R., Ho, I.-T., et al. 2014, ApJ, 795, L37

Cortese, L., Fogarty, L. M. R., Bekki, K., et al. 2016, MNRAS, 463, 170

Cortese, L., van de Sande, J., Lagos, C. P., et al. 2019, MNRAS, 485, 2656

Crain, R. A., Schaye, J., Bower, R. G., et al. 2015, MNRAS, 450, 1937

Croom, S. M., Lawrence, J. S., Bland-Hawthorn, J., et al. 2012, MNRAS, 421, 872

Davies, R. L., Efstathiou, G., Fall, S. M., et al. 1983, ApJ, 266, 41

Davis, T. A., \& Bureau, M. 2016, MNRAS, 457, 272

de Zeeuw, P. T., Bureau, M., Emsellem, E., et al. 2002, MNRAS, 329, 513

de Zeeuw, T., \& Franx, M. 1991, ARA\&A, 29, 239

Driver, S. P., Hill, D. T., Kelvin, L. S., et al. 2011, MNRAS, 413, 971

Dubois, Y., Pichon, C., Welker, C., et al. 2014, MNRAS, 444, 1453

Emsellem, E., Cappellari, M., Krajnović, D., et al. 2011, MNRAS, 414, 888

Falcón-Barroso, J., Lyubenova M., \& van de Ven, G. 2015, IAUS, 78, 311

Green, A. W., Croom, S. M., Scott, N., et al. 2018, MNRAS, 475, 716

Greene, J. E., Leauthaud, A., Emsellem, E., et al. 2017, ApJ, 851, L33

Hirschmann, M., Dolag, K., Saro, A., et al. 2014, MNRAS, 442, 2304

Illingworth, G. 1977, ApJ, 218, L43

Kassin, S. A., Weiner, B. J., Faber, S. M., et al. 2007, Deepest Astronomical Surveys, 477

Krajnović, D., Emsellem, E., Cappellari, M., et al. 2011, MNRAS, 414, 2923

Lange, R., Moffett, A. J., Driver, S. P., et al. 2016, MNRAS, 462, 1470 
Ma, C.-P., Greene, J. E., McConnell, N., et al. 2014, ApJ, 795, 158

Naab, T., Oser, L., Emsellem, E., et al. 2014, MNRAS, 444, 3357

Owers, M. S., Allen, J. T., Baldry, I., et al. 2017, MNRAS, 468, 1824

Robotham, A. S. G., Norberg, P., Driver, S. P., et al. 2011, MNRAS, 416, 2640

Sánchez, S. F., Kennicutt, R. C., Gil de Paz, A., et al. 2012, A\&4A, 538, A8

Schaye, J., Crain, R. A., Bower, R. G., et al. 2015, MNRAS, 446, 521

Scott, N., van de Sande, J., Croom, S. M., et al. 2018, MNRAS, 481, 2299

Sharp, R., Saunders, W., Smith, G., et al. 2006, Proc. SPIE, 62690G

Sharp, R., Allen, J. T., Fogarty, L. M. R., et al. 2015, MNRAS, 446, 1551

Tinsley, B. M. 1980, Fundamentals Cosmic Phys., 5, 287

van de Sande, J., Bland-Hawthorn, J., Fogarty, L. M. R., et al. 2017, ApJ, 835, 104

van de Sande, J., Scott, N., Bland-Hawthorn, J., et al. 2018, Nature Astronomy, 2, 483

van de Sande, J., Lagos, C. D. P., Welker, C., et al. 2019, MNRAS, 484, 869

Veale, M., Ma, C.-P., Greene, J. E., et al. 2017, MNRAS, 471, 1428

Weiner, B. J., Willmer, C. N. A., Faber, S. M., et al. 2006, ApJ, 653, 1027 